\title{
AUFSÄTZE
}

\section{Innovationen und Finanzmarkt}

Hartmut Hirsch-Kreinsen

Sowohl technologische Innovationen als auch der Finanzmarkt sind zentrale Bestimmungsgrößen der ökonomischen Entwicklung. Beide Faktoren stehen in einem engen Zusammenhang. Insbesondere die Finanzmarktdynamik der letzten Jahre legt die Frage nahe, welche Konsequenzen sich daraus für das deutsche Innovationssystem ergeben. Es zeigt sich, dass die Finanzmarktorientierung vieler Unternehmen zu einer verschärften Ökonomisierung von Innovationsprozessen und zu einer Beschränkung von zuvor vorhandenen Innovationsspielräumen führt. Bisherige technologisch anspruchsvolle Innovationen kommen zunehmend unter ökonomischen Druck, jedoch eröffnen sich zugleich auch andere Innovationsmöglichkeiten in bislang vernachlässigten Technologiebereichen.

\section{1 \\ Vorbemerkung}

Die Fragestellung des Beitrages richtet sich auf den Zusammenhang zwischen den Verlaufsmustern technologischer Innovationen und der Dynamik des Finanzmarktes, wobei die Entwicklung des deutschen Innovationssystems im Fokus steht. Die Analyseperspektive zielt folglich auf das $\mathrm{Zu}-$ sammenspiel zweier zentraler Strukturbedingungen und Entwicklungsmomente kapitalistischer Gesellschaften. Unumstritten ist in der sozialwissenschaftlichen Debatte, dass Innovationen ein wesentliches treibendes Moment der ökonomischen Dynamik sind. Als die zentralen Akteure, die Innovationen generieren und vorantreiben, gelten dabei Unternehmen. Unumstritten ist in der Innovationsforschung aber auch, dass Verlauf und Reichweite von technologischen Innovationen in Wechselwirkung mit den je gegebenen gesellschaftlich-institutionellen Bedingungen stehen. Dabei wird zunächst davon ausgegangen, dass stets das Zusammenspiel der Gesamtheit der verschiedenen institutionellen Faktoren ein Innovationssystem und die darin verankerten Unternehmensstrategien prägen (zusammenfassend: Werle 2005). Zugleich aber gilt der Finanzmarkt insofern als wesentliche Strukturbedingung der kapitalistischen Produktionsweise, da auf diesem über Bedingungen und Volumen der Allokation von Kapital für wirtschaftliche Aktivitäten entschieden wird (Hall/ Soskice 2001; Lane 2003). Freilich ist dabei nicht der Finanzmarkt als Ganzes von In- teresse, sondern nur jene Segmente, in deren Kontext die Fremd- und Eigenfinanzierung von Unternehmen, von Regierungen und von Privatpersonen geregelt wird. Wenn im Folgenden vom Finanzmarkt die Rede ist, so geht es um diese letztgenannten Segmente der Unternehmens- und Innovationsfinanzierung. ${ }^{1}$

$\mathrm{Zu}$ diesem, schon von Joseph Schumpeter für die wirtschaftliche Entwicklung zentral gestellten Zusammenhang zwischen Finanzmarktbedingungen einerseits und Innovationsstrategien von Unternehmen andererseits (Schumpeter 1997, S. 99ff.) gibt es aktuell nur wenige sozialwissenschaftliche Analysen. ${ }^{2}$ Dieses Forschungsdefizit greift die folgende Argumentation auf: Erstens werden strukturelle Probleme der Innovationsfinanzierung diskutiert. Zweitens wird skizziert, in welcher Weise diese Finanzierungsprobleme im deutschen Innovationssystem traditionell bewältigt werden. Drittens werden die Konsequenzen der in den letzten Jahren beobachtbaren Internationalisierung des Finanzmarktes und der Unternehmensfinanzierung für Innovationsprozesse erörtert. Und schließlich werden Thesen zu Entwicklungstendenzen des deutschen Innovationssystems präsentiert. Die Argumentation basiert primär auf einer Auswertung von Studien, die sich direkt oder auch nur indirekt mit dieser Fragestellung befassen, der Aufarbeitung aktueller innovationspolitischer $\mathrm{Pu}$ blikationen sowie einigen empirischen Evidenzen. Insgesamt werden damit Überlegungen aus einer laufenden Arbeit zur Diskussion gestellt.

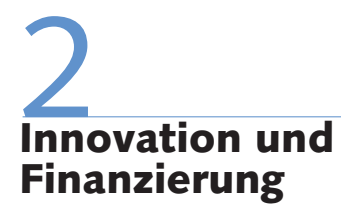

Im Folgenden sollen mit dem Begriff der Innovation im Anschluss an die sozialwissenschaftliche Innovationsforschung technologische Innovationen angesprochen werden, nämlich die Genese, Entwicklung und Diffusion neuer marktgängiger Produkte, Dienstleistungen und technisch-organisatorischer Verfahren (z. B. Fagerberg 2005). Die hervorstechenden Merkmale technologischer Innovationen sind ihre Ungewissheit im Hinblick auf den erreichbaren technischen und ökonomischen Erfolg, die Risiken des in der Regel immer nur begrenzt vorausplanbaren Ablaufs des Innovationsprozesses mit seinen nur schwer ex ante kalkulierbaren Zwischenschritten und un-

\footnotetext{
1 Davon zu unterscheiden sind jene Finanzmarktsegmente, in denen Finanzforderungen und Zahlungsverpflichtungen über Währungen, Anleihen, Derivate etc. gehandelt werden (Knorr Cetina/ Preda 2006).

2 Zum Stand der Forschung vgl. O'Sullivan (2005) und Tylecote/Visintin (2009, S. 4f.).
}

Hartmut Hirsch-Kreinsen, Prof. Dr., lehrt Wirtschafts- und Industriesoziologie an der TU Dortmund. Arbeitsschwerpunkte: Innovationsprozesse, Entwicklungstendenzen von Arbeit, Unternehmensnetzwerke. e-mail: hartmut.hirsch-kreinsen@tudortmund.de 
erwartet auftretenden Entscheidungssituationen sowie schließlich die nur schwer kalkulierbaren Innovationskosten. Ein weiteres zentrales Merkmal technologischer Innovationen ist der häufig nur immaterielle Charakter ihrer Voraussetzungen, vor allem ihrer Wissensbasis. So betont die Innovationsforschung, dass erfolgreiche Innovationen in hohem Maße auf nicht-kodifizierbarem Erfahrungswissen basieren (Dosi 1990).

Versucht man den wechselseitigen $\mathrm{Zu}$ sammenhang zwischen Innovationen und Finanzierung genauer zu bestimmen, so ist von einem strukturellen Spannungsverhältnis zwischen beiden auszugehen. Denn zum einen stehen Risiken und Ungewissheit von Innovationen dem Interesse von Investoren an Sicherheit ihrer Anlagen und der Kalkulierbarkeit der Rendite entgegen. Zum anderen stellt sich das Problem der asymmetrischen Informationen zwischen dem Innovator und dem Finanzier, was zu Opportunismus auf der Seite des Innovators und Fehlentscheidungen auf der Seite des Finanziers führen kann. Denn in jedem Fall verfügt der Innovator über ein weit besseres Verständnis der Möglichkeiten, Risiken und Unsicherheiten eines Innovationsvorhabens als ein externer Finanzier (Gerybadze 2004, S. 290ff.). Die Innovationsforschung spricht daher auch vom Innovationsdilemma. Einerseits wirke sich eine strikte Unterordnung von Innovationsaktivitäten unter ökonomisches Kalkül wie unter die Interessen externer Finanziers als Hemmnis von Innovationen aus und es kämen nur Ketten von Kleinund Scheininnovationen zustande. Andererseits erweise sich eine weitreichende Autonomisierung von Innovationsprozessen gegenüber ökonomischen Kriterien der Effizienz und Rentabilität als kostenverschlingendes und bestandsgefährdendes Verwertungsrisiko (Rammert 1992, S. 11).

\section{Das deutsche "Insider-dominierte" Innovationssystem}

Wie Forschungsergebnisse zeigen, werden diese Finanzierungsprobleme von Innovationen historisch und nationalspezifisch auf unterschiedlichem Wege bewältigt, womit sich ebenso unterschiedliche Innovationsverläufe verbinden (Werle 2005). Die für die deutsche Industrie typischen technologisch anspruchsvollen inkrementellen Innovationen in Industrien wie Chemie, Elektrotechnik, Maschinenbau und Fahrzeugbau stehen im Kontext eines Systems der Unternehmensfinanzierung, das sich neben der Eigenfinanzierung der Unternehmen aus Gewinnen, Abschreibungen und weiteren Komponenten des CashFlow vor allem durch Formen der Kreditfinanzierung und eine damit verbundene Langfristorientierung der Kreditgeber auszeichnet. Daraus ergibt sich eine spezifische Form ökonomischer Rationalität: Die kreditgebenden Banken sind nicht daran interessiert, dass ihre Schuldner, d.h. die Unternehmen, kurzfristige Strategien der Profitmaximierung verfolgen und dabei Risiken im Hinblick auf ihre langfristige Existenz eingehen. Denn nur wenn solche vermieden werden, sind Tilgung und Rückzahlung der Kredite gewährleistet und die Profite der Gläubiger-Bank gesichert (Windolf 2005). Die relativ hohe Bedeutung der Kreditfinanzierung zeigt sich daran, dass in dem Zeitraum zwischen 1975 und 1995 Bankkredite ein knappes Fünftel der gesamten Unternehmensfinanzierung ausmachten (Huffschmid 1999, S. 19); bei mittleren und kleinen Unternehmen des Verarbeitenden Gewerbes betrug Mitte der 1990er Jahre die Finanzierung über langfristige und kurzfristige Verbindlichkeiten bei Banken sogar bis zu $60 \%$ der Gesamtfinanzierung (Vieweg 2001, S. 53).

Der institutionelle Rahmen dieses Finanzierungssystems ist der eines vernetzten von Universalbanken und industriellen Überkreuzbeteiligungen bestimmten Systems der Corporate Governance. Vorherrschend sind Eigentümer- und Kontrollstrukturen, die in international vergleichenden Studien von Systemen der Unternehmensfinanzierung als „Insider-System “ bezeichnet werden. ${ }^{3}$ Seine hervorstechenden Merkmale sind ein hohes unternehmensorientiertes Engagement von unternehmensexternen Kapitalgebern wie auch deren relativ intime Kenntnis der Situation und der Aktivitäten der von ihnen finanzierten Unternehmen (Dosi 1990; Tylecote/ Ramirez 2006). Im Hinblick auf Innovationsprozesse impliziert dies eine enge und längerfristige Bindung externer Kapitalgeber an ein innovierendes Unternehmen auf der Basis einer relativ genauen Kenntnis der zu finanzierenden Prozesse. Giovanni Dosi (1990) argumentiert, dass dauerhafte Lernprozesse zwischen den beteiligten Ak- teuren für die hier stattfindenden Innovationen charakteristisch seien, wobei die Rolle der Investoren auf Beteiligung und Einflussnahmen basiere und ihr Zugriff auf unternehmensinterne Finanzmittel begrenzt sei. Unternehmensintern entspricht dieser Situation ein Managementtypus, der einen sukzessiven und langfristig orientierten Aufstieg innerhalb des Unternehmens absolviert und daher eine genaue Kenntnis der technologischen Prozesse aufweist (Freye 2009). Vielfach ist die Voraussetzung für eine solche Hauskarriere ein technischnaturwissenschaftliches Examen, typisch ist der akademische Abschluss eines Diplomingenieurs.

Die Folge ist, dass in diesem Rahmen Innovationen mit langfristiger Perspektive schrittweise entlang gegebener technologischer Entwicklungspfade verlaufen. Denn die akkumulierten Kompetenzen der beteiligten unternehmensexternen und -internen Akteure, die eingespielten organisatorischen Routinen und die getätigten Investitionen im Kontext der langfristig angelegten Beziehungen der beteiligten Akteure stehen radikalen Innovationen und ihren Risiken und Unsicherheiten entgegen. Die oben angeführte Debatte über Finanzierungssysteme paraphrasierend, lässt sich daher das traditionelle deutsche Innovationssystem auch als Insider-dominiert bezeichnen. Nicht überraschend ist, dass in diesem Kontext Unternehmen, die an radikalen Innovationen arbeiten, im Nachteil sind. Denn angesichts der vorherrschenden Finanzierungsmodalitäten ist risikoorientiertes Venture Capital für solche Innovationsstrategien nur begrenzt verfügbar (vgl. z. B. Caspar et al. 1999).

\section{1 \\ Internationalisierung des Finanzmarktes}

Nun macht die neuere wirtschaftssoziologische Forschung deutlich, dass infolge der Internationalisierung der Finanzmärkte eine Tendenz zur Auflösung der vernetzten Strukturen der „Deutschland-AG“ unübersehbar ist und von einem neuen Produktionsregime gesprochen werden kann, das

3 Demgegenüber wird das finanzmarktorientierte System der USA als "Outsider-System" gekennzeichnet (Franks/Mayer 1997). 
als „Finanzmarktkapitalismus“ zu bezeichnen ist (Windolf 2005, 2008). Das von relativ autonomen Universalbanken geprägte vernetzte System der Vergabe langfristiger Kredite werde tendenziell von einem international orientierten, sehr stark auf angelsächsischen Regeln des Finanzmarktes und der Unternehmensfinanzierung basierenden System abgelöst (Höpner 2003; Beyer 2006; Kädtler 2005).

Die Frage ist nun, in welcher Weise diese Veränderungen das „Insider-dominierte" Innovationssystem und die darin verankerten Innovationsstrategien von Unternehmen beeinflussen. Endgültige Antworten sind derzeit kaum möglich, doch erlauben die wenigen vorliegenden und recht disparaten Forschungsergebnisse einige begründete Annahmen über ebenso neuartige wie widersprüchliche Entwicklungstrends. Einerseits zeigt sich auf einer aggregierten Ebene, dass die Aufwendungen der deutschen Unternehmen für Forschung und Entwicklung (FuE) gerade auch in den letzten Jahren gestiegen sind. ${ }^{4}$ Andererseits geben die Forschungsergebnisse einige instruktive Hinweise auf strukturelle Veränderungstendenzen, die sich je nach Segment des Finanzmarktes, dem jeweiligen Innovationstypus ${ }^{5}$ und dem gegebenen System der Corporate Governance 6 eines Unternehmens unterschiedlich durchsetzen.

\subsection{BESCHRÄNKUNG VON INNOVATIONSSPIELRÄUMEN}

Folgt man den vorliegenden Forschungsergebnissen, so sind Tendenzen einer Erosion des bisherigen Innovationssystems, verbunden mit einer Beschränkung der bisherigen Innovationsspielräume von Unternehmen, unübersehbar. Damit kann eine von Christoph Deutschmann (2005, 2008) und William Lazonick $(2003,2007)$ vertretene Hypothese aufgegriffen werden. Danach seien unter den geänderten Finanzierungsbedingungen, insbesondere aufgrund massiv erhöhter Rentabilitätserwartungen und einer ausgeprägten Kurzfristorientierung von Investoren wie Pensions- und Investmentfonds sowie Hedge-Fonds, weder eine hinreichende Stabilität der finanziellen Mittel noch ausreichende strategische Handlungsspielräume für die Unternehmen gegeben. Zudem seien die dominanten Finanzmarktakteure und die neuen Finanzierungsregeln wie auch Instrumente aufgrund fehlender Kontextbezüge nicht in der Lage, die Risiken und Unsicherheiten von technischen Innovationen sowie ihre unternehmensstrukturellen Voraussetzungen hinreichend einzuschätzen. Als eine Konsequenz wird gesehen, dass kollektive Lernprozesse und kumulative Wissensakkumulation begrenzt und Innovationsvorhaben auf ökonomisch planbare Aktivitäten reduziert werden.

\section{AUSRICHTUNG AUF ÖKONOMISCHE KRITERIEN}

Erkennbar wird diese Tendenz im Kapitalmarktsegment mit börsennotierten Firmen und einer engen Kopplung an die Entscheidungskriterien und die Interessen relevanter Finanzmarktakteure. Ein Beispiel hierfür sind große Pharmaunternehmen, die in den letzten Jahren ihre Wertschöpfungsketten, insbesondere die Forschungsbereiche, weitreichend reorganisiert haben, um sich auf ihre als Kernkompetenzen erachteten Funktionen zu konzentrieren und dadurch den Unternehmenswert zu steigern. Resümiert man die hierzu vorliegenden Forschungsergebnisse, so lässt sich zweierlei festhalten (Briken/Kurz 2006; Kädtler 2009): Zum einen findet eine deutliche Straffung der Innovationsprozesse und ihre strikte Steuerung nach Finanzkennziffern und weiteren Indikatoren statt, um Fehlentwicklungen frühzeitig zu erkennen und Entwicklungszeiten zu verkürzen. Zum zweiten verbindet sich mit der Bindung der Innovationsprozesse im Pharmabereich an Finanzmarktkriterien eine Fokussierung der Innovationsstrategien auf besonders aussichtsreiche Produkte, sogennante Blockbuster, d.h. patentgeschützte, umsatz- und margenträchtige Schlüsselprodukte. Freilich begründet sich dieser Innovationsfokus in einer letztlich risikoaversen Vorliebe für die nur begrenzte Modifikationen im Prinzip bekannter chemischer Einheiten. ${ }^{7}$ Durchaus ähnlich lässt sich die Innovationsentwicklung in der Automobilindustrie interpretieren, wo unter den Bedingungen einer ausgeprägten Innovationskonkurrenz Rentabilität über steigende und sichtbare Innovationen generiert werden soll (Tylecote/Visintin 2009, S. 40ff.). Merkmale der hohen Innovationsdynamik sind eine seit den 1990er Jahren deutliche Erhöhung des gesamten Aufwandes für FuE, die starke Verkürzung der Entwicklungszeiten und die massive Verbreiterung des Produktangebots (EFI 2009, S. 81). Zugleich jedoch sind einige große Automobilunternehmen Vorreiter im Hinblick auf finanzmarktorientierte Unternehmensstrategien. Dies lässt sich vor allem an der Strategie festmachen, die Unternehmensstrukturen auf die sogenannte Kernkompetenzen zu beschränken und die Fertigungstiefe kontinuierlich abzubauen. Folgen dieser Maßnahmen sind eine seit Mitte der 1990er Jahre kontinuierliche Reduktion der unternehmensinternen FuE-Aufwendungen und ein starker $\mathrm{Zu}$ wachs der externen FuE-Aufwendungen, womit das Innovationsrisiko insbesondere bei der Nutzung neuer Technologien auf externe Zulieferunternehmen verlagert wird. Insgesamt fokussierten sich die Innovationsanstrengungen der letzten Jahre besonders auf etablierte Technologiepfade und komplexe Produkte, mit denen kurzfristig hohe Umsätze und Margen gesichert werden konnten, während neue Technologien z. B. im Antriebsbereich vernachlässigt wurden (Jürgens/Sablowski 2008, S. 103ff.).

Ähnliche Konsequenzen hat offensichtlich die Übernahme von Firmen durch nicht-öffentliche Beteiligungsgesellschaften, namentlich Private Equity-Fonds. Deren Strategie richtet sich auf die kreditfinanzierte Beteiligung an Unternehmen, die Ausschöpfung der finanziellen Potenziale übernommener Unternehmen und einen schnellen Ausstieg aus Beteiligungen zu einem möglichst hohen Verkaufswert zugespitzt in der Formel ,invest to sell“ (Klier et al. 2009). Folgt man der vorliegenden Literatur (zusammenfassend z. B. Kamp 2007), so kann sich kurzfristig mit diesen Übernahmen durchaus eine Steigerung der Effizienz und Wettbewerbsfähigkeit der Unternehmen verbinden,

4 Im internationalen Vergleich stieg der FuE-Aufwand deutscher Unternehmen allerdings unterdurchschnittlich, zudem blieb er stets hinter der Umsatzentwicklung zurück (EFI 2009, S. 78).

5 Angesprochen sind hiermit die Reichweite einer Innovation (z. B. inkrementell vs. radikal), der Innovationsgegenstand (Produkt- oder Prozessinnovation), das technologische Feld und der Grad der Sichtbarkeit wie aber auch die mögliche Verwertbarkeit der Innovationserträge (Tylecote/Ramirez 2006).

6 Mit dem Begriff des Systems der Corporate Governance wird der Komplex von Strukturen, Interessen und Praktiken verstanden, durch die Unternehmen und ihre Strategien gesteuert und kontrolliert werden.

7 Auf diesen Aspekt wurde der Autor von einem anonymen Gutachter der WSI-Mitteilungen hingewiesen. 
längerfristig orientierte Strategien und Investitionen in Forschung und Entwicklung sind jedoch kaum mehr möglich. Dies belegt eine ganze Reihe bekannt gewordener Übernahmefälle dieser Art, beispielsweise von mittelständischen Unternehmen aus der Investitionsgüterindustrie: Die Investoren scheuen das finanzielle Risiko von Innovationen, sie bringen wenig Verständnis für diese auf und aufgrund der oft durchgreifenden Restrukturierungsmaßnahmen mit der Konsequenz eines Abbaus von vermeintlich überschüssigen Ressourcen und einer Reduktion der Unternehmensfunktionen auf Kernkompetenzen gehen für Innovationen oft unverzichtbare organisatorische Spielräume und personelle Kompetenzen verloren (z. B. Lembke 2008).

\section{VERSCHÄRFTE BEDINGUNGEN FÜR KREDITVERGABE}

Diese ökonomisch restriktive Situation für Innovationsvorhaben wird durch neue Regularien der externen Unternehmensfinanzierung verstärkt. Zu nennen sind hier die Effekte neuer Regeln der Kreditvergabe (sogenannte Basel-II-Regelungen) und verschärfte Bonitätsprüfungen der Unternehmen mit ihren risikoaversen und aufwendigen Rating- und Bewertungsverfahren, die Innovationsvorhaben einer ausgeprägteren und systematischeren ökonomischen Kontrolle als früher unterwerfen (KfW-Research 2006; Springler 2007). Diesen Finanzierungsrisiken sind zum einen Unternehmen ausgesetzt, die wegen begrenzter Eigenmittel Innovationsaktivitäten zu einem großen Anteil mit zweckgebundenen Bankkrediten finanzieren müssen. Zum zweiten berührt dies Unternehmen mit wenig sichtbaren Produktinnovationen und solche mit Prozessinnovationen, die bislang Kredite zur Finanzierung nutzten (Rammer 2009, S. 48ff.). Insgesamt dürfte es sich dabei vor allem um Unternehmen aus technologisch traditionellen Branchen handeln, deren Innovationsstrategien sich besonders auf inkrementelle Produkt- und Prozessinnovationen richten (Hirsch-Kreinsen 2008). Daneben sind generell kleinere und mittlere Unternehmen (KMU) diesen Restriktionen unterworfen, da sich ihre Innovationsaktivitäten im Vergleich zu denen von Großunternehmen durch ,stärker idiosynkratische Risiken“" auszeichnen, die aus einer Spezialisierung auf Nischenmärkte und kundenspezifische Lösungen resultieren (KfW-Research 2006, S. 108). Diese Bedingungen erhöhen aus der Sicht externer Kapitalgeber die unkalkulierbaren Risiken und Erfolgsaussichten solcher Innovationsvorhaben, sodass sich eine unternehmensexterne Innovationsfinanzierung für KMU häufig nur zu hohen Kosten oder, etwa aufgrund mangelnder Projektrentabilität, gar nicht realisieren lässt (Rammer et al. 2006, S. 124ff.).

Die Erosion des Insidersystems ist allerdings nicht allein auf den wachsenden Einfluss der Finanzmarktakteure zurückzuführen. Wie Jürgen Kädtler (2005) instruktiv zeigt, kommen als verstärkender Faktor finanzmarktorientierte Handlungsorientierungen des Topmanagements vieler Unternehmen ins Spiel, die unabhängig vom tatsächlichen Einfluss des Finanzmarktes und seiner Akteure eine Wirkung entfalten. Ein Grund hierfür sind die viel diskutierten Aktien-Optionen und Bonisysteme, die Managern bei einer gelungenen finanzmarktorientierten Unternehmensreorganisation massive Einkommenszuwächse bescheren. Ein weiterer Grund ist, dass sich in vielen Unternehmen der deutschen Industrie seit den 1990er Jahren ein neuer Managertyp durchsetzt, der den oben erwähnten Typus des zumeist technischnaturwissenschaftlichen Spezialisten mit einer langjährigen Unternehmensbindung ablöst. Demgegenüber finden sich nun vermehrt allgemein qualifizierte Generalisten und Manager aus dem Finanz- und Controllingbereich auf den entsprechenden Stellen, und die Fluktuation bzw. die Mobilität der Topmanager erhöht sich deutlich (Beyer 2006; Freye 2009). Die Konsequenzen für Innovationsstrategien von Unternehmen liegen auf der Hand: Die genaue Kenntnis und Vertrautheit mit technologischen Entwicklungspotenzialen nimmt ab und risikoreiche Innovationen werden vermieden. Weiterhin konterkariert die kurzfristige Karriereperspektive vieler Manager die für Innovationen vielfach erforderliche Langfristorientierung.

\subsection{BEHARRUNGSKRÄFTE UND GEGENTENDENZEN}

Allerdings verweist auch eine Reihe von empirischen Befunden auf Beharrungskräften und Gegentendenzen gegen die Erosion des bisherigen Innovationssystems und die Beschränkung von Innovationsspielräumen.

\section{BEHARRUNGSKRÄFTE}

Als Ausdruck für Beharrungskräfte kann die nach wie vor hohe Bedeutung unternehmensinterner Finanzierungsquellen für Innovationen interpretiert werden. Daten des Zentrums für europäische Wirtschaftsforschung (ZEW) Mannheim (Rammer 2009) aus den Jahren 2004-2006 zeigen, dass ein großer Teil der innovierenden Unternehmen zur Innovationsfinanzierung ausschließlich auf unternehmensinterne Mittel aus dem laufenden Geschäftsbetrieb zurückgreift. Ein anderer, ebenso großer Teil der Unternehmen kombiniert interne mit externen Finanzierungsquellen; die genauen Zahlen lauten: $82 \%$ aller Unternehmen greifen auf interne Finanzquellen, die sich aus dem laufenden Geschäftsbetrieb ergeben (Cash-Flow), zurück; davon nutzen $41 \%$ der Unternehmen diese ausschließlich, während weitere $41 \%$ sie mit externen Mitteln kombinieren. Eine andere Form der internen Innovationsfinanzierung sind Gesellschafterdarlehen, die von rund $18 \%$ aller Unternehmen genutzt werden. Insgesamt findet sich diese Innovationsfinanzierung aus Eigenmitteln vor allem bei Unternehmen, die eine vergleichsweise hohe FuE-Intensität aufweisen und relativ risikoreiche, zumindest von außen schwer kalkulierbare Innovationsaktivitäten durchführen. Konkret findet sich diese Finanzierungssituation beispielsweise im mittelständischen Maschinenbau (Rammer 2009, S. 41ff.). Klammert man an dieser Stelle den möglichen Einfluss finanzmarktorientierter Leitbilder der neuen Generalisten im Management einmal aus, so ist davon auszugehen, dass die Unternehmen sich durch die interne Finanzierung eigene Handlungsspielräume für risikoreiche und weitreichende Innovationsvorhaben sichern und externe Finanzierungsrisiken zu umgehen suchen.

Darüber hinaus sind Beharrungskräfte zu sehen, die in den strukturellen Bedingungen der Innovationsprozesse selbst angelegt sind: Wie arbeitssoziologische Studien überzeugend belegen (z. B. Wolf et al. 1992; Kalkowski et al. 1995) kann Innovationsarbeit wegen der stets auftretenden Risiken und Ungewissheiten kaum durchgreifend kosten- und rentabilitätsorientiert standardisiert werden, da sich die funktionale Logik dieser Arbeitsprozesse vielfach solchen Versuchen sperrt. Denn für das Ergebnis von Innovationsarbeit ist oft ein „Eigenbeitrag“ der Wissenschaftler, Inge- 
nieure und Techniker unabdingbar, der auf erfahrungsgesättigten Primärinformationen über Entwicklungsprobleme und ihrer Bewältigung beruht. Hinzu kommt, dass sich neue Innovationspotenziale oftmals ungeplant ergeben und ihre Bedeutung für Neuentwicklungen allein „vor Ort" kompetent eingeschätzt und genutzt werden kann. Als Beispiel hierfür sei auf ein erfolgreiches Pharmaprodukt verwiesen, das nur zustande kam, weil zwei Entwickler ein offiziell schon abgebrochenes Entwicklungsprojekt unter der Hand weiterbetrieben haben (Kädtler 2009, S. 17). Dies gilt insbesondere auch dann, wenn durch neue Organisations- und Managementkonzepte oder durch die Einführung rechnergestützter Steuerungs- und Controllingsysteme die Innovationsprozesse gestrafft werden sollen. Denn sie bedürfen der störungsfreien Anpassung an die je konkreten Erfordernisse, die angesichts der stets auftretenden Risiken und Ungewissheiten nur auf der operativen Ebene von den Experten selbst erbracht werden kann. Voraussetzung hierfür ist die „Aneignung“ der jeweiligen Systeme, d.h. ihre kompetente Anpassung an die gegebenen Bedingungen durch ihre Nutzer. Insgesamt resultieren daraus nicht nur strukturelle Grenzen der Rationalisierbarkeit von Innovationsprozessen, sondern auch ein beträchtliches Machtpotenzial der betroffenen Forscher und Entwicklungsingenieure, das sie in die Lage versetzt, Kennziffersysteme und Benchmarkingverfahren in ihrem Interesse zu beeinflussen.

\section{GEGENTENDENZEN}

Gegentendenzen gegen die Einschränkung von Innovationsspielräumen ergeben sich im Zusammenhang mit dem wachsenden Einfluss von technologie- und innovationsorientierten Kapitalanlegern. Zum einen sind an dieser Stelle Investoren wie einige industriell orientierte Private EquityFonds $\mathrm{zu}$ nennen, die eine langfristig orientierte Anlagestrategie verfolgen und Unternehmen neue Handlungsspielräume für Produktivitätssteigerungen und Innovationen eröffnen (Achleitner et al. 2008). Zum zweiten spielt hier Wagniskapital eine wichtige Rolle, das sich auf die Finanzierung von risikoreichen Produktinnovationen im Hightech-Bereich richtet. Der grundlegende Mechanismus dieser Finanzierungsform ist, das ein Wagniskapitalgeber oft auf der Basis einer sehr genauen
Kenntnis des jeweiligen Technologiefelds und mit hoher Risikobereitschaft in Innovationsvorhaben investiert und an einem möglichst raschen Rückfluss hoher Gewinne, sei es durch die erfolgreiche Vermarktung eines neuen Produktes, sei es durch den Verkauf seiner Firmenanteile, interessiert ist. Die hohe Risikobereitschaft der Anleger resultiert nicht zuletzt daraus, dass solche Investitionen stets nur einen begrenzten Teil ihres gesamten Anlageportfolios ausmachen und damit die Anlagerisiken insgesamt begrenzt bleiben. Grundsätzlich jedoch konvergieren in diesem Finanzierungssegment die spekulativen und auf kurzfristige Gewinne gerichteten Motive der Investoren mit den risikoreichen, aber auch sehr gewinnträchtigen Perspektiven von radikalen Innovationen in neuen Technologiefeldern.

Sieht man einmal von dem New-Economy-Boom der zweiten Hälfte der 1990er Jahre ab, so spielt im Vergleich besonders zu den USA Wagniskapital in seinen verschiedenen Ausprägungen in Deutschland insgesamt gesehen eine nachgeordnete Rolle für die Finanzierung von Innovationen (BMBF 2007). In einigen Hochtechnologiebranchen kommt Wagniskapital jedoch auch in Deutschland ein größerer Stellenwert zu. Dies betrifft vor allem die IT- und Biotechnologie-Branche wie auch die Medizintechnik, die Automatisierungs-, Steuer- und Regelungstechnik sowie die Kommunikationstechnik. Schätzungen zufolge sind in der Biotechnologiebranche ein Fünftel bis ein Viertel der FuE-Ausgaben durch Wagniskapital finanziert (KfWResearch 2006, S. 121; Champenois et al. 2006). ${ }^{8}$ Ein oft unterschätztes Teilsegment des Marktes für Wagniskapital wird zudem von sogenannten Business Angels gebildet. Dabei handelt es sich um vermögende und hoch spezialisierte Privatpersonen, die in der Lage sind, die Risiken und Unsicherheiten von speziellen Innovationen zureichend einzuschätzen und den innovierenden Unternehmen entsprechende Finanzierungsmöglichkeiten, aber auch Beratungskompetenzen anzubieten (Carpenter et al. 2003; Tylecote/Ramirez 2006). Obgleich bislang nur Daten vorliegen, die auf Schätzungen beruhen, ist auch dieses Segment des Finanzmarktes in Deutschland insgesamt im internationalen Vergleich sehr klein, jedoch für bestimmte Hochtechnologieunternehmen, insbesondere sehr forschungsintensive Spin-offs aus dem Wissenschaftsbereich, ebenfalls über- aus bedeutsam (Fryges et al. 2007). Auch ist auf ein weiteres Teilsegment des Marktes für Wagniskapital in diesem Zusammenhang hinzuweisen: das sogenannte Corporate Venture Capital. Damit finanzieren Großunternehmen über eigens gegründete Gesellschaften für sie interessante Innovationsvorhaben anderer kleinerer Unternehmen. Die Finanzierungsstrategie ist langfristig orientiert und in der Regel gekoppelt mit einer engen Beratung des innovierenden Unternehmens. Hauptsächlich findet sich diese Finanzierungsform in der Pharmabranche. Trotz gegenwärtiger Krise wächst dieses kleine Finanzmarktsegment aufgrund des hohen Innovationsdrucks in der Pharmabranche erkennbar (FAZ 2009).

Grundlegendes Merkmal dieser Finanzierungsformen von Innovationen ist die häufig enge personelle Verflechtung zwischen innovierenden Firmen und den Kapitalgebern. Auf der Basis ihrer genauen Kenntnis eines bestimmten Technologiefeldes sind die Investoren häufig eng mit der finanzierten Firma verbunden und beteiligen sich in verschiedenen leitenden Funktionen innerhalb des Unternehmens am laufenden Geschäft. Insgesamt ist daher die Kontrolle der Firmenleitung durch die Wagniskapitalgeber enger als im Fall anderer Formen der externen Innovationsfinanzierung (Rammer 2009, S. 37). Festhalten lässt sich, dass ganz im Gegensatz zu den skizzierten Erosionstendenzen des deutschen Insider-dominierten Innovationssystems auf dem Segment des Wagniskapitals eine Neukonturierung des Insider-Systems beobachtbar ist. Sein zentrales Merkmal ist eine enge Bindung der innovierenden Unternehmen an Finanzmarktakteure und ihr Interesse an kurzfristigen und hohen Gewinnen, die durch einen zugleich engen Bezug der Investoren auf die technologischen Erfordernisse, Risiken und Ungewissheiten eines Innovationsprozesses realisiert werden sollen. Anders formuliert: Finanzmarktakteure agieren in diesem Fall als technologische Insider, um auf diesem Wege ihre Anlageinteressen zu realisieren.

\footnotetext{
8 Dabei sind die Finanzierungsvolumina ausländischer Beteiligungsgesellschaften, die in dem Sektor der Biotechnologie eine wichtige Rolle spielen, nicht berücksichtigt (KfW-Research 2006, S. 121).
} 


\section{Entwicklungsperspektiven}

Insgesamt führen die skizzierten Tendenzen zu einer Ausdifferenzierung des bisherigen, relativ homogenen Insider-dominierten Innovationssystems in Deutschland: Einerseits erodieren unter dem Einfluss des internationalisierten Segments des Kapitalmarktes wesentliche Bereiche des bisherigen Systems mit der Folge einer verschärften Ökonomisierung von Innovation und einer Beschränkung bisheriger Innovationsspielräume. Zugespitzt formuliert: Der bislang dominierende Einfluss von technologieorientierten „Insidern“ wird abgelöst von finanzmarktorientierten „Outsidern“. Andererseits eröffnen sich, forciert durch das Segment des Wagniskapitals, neue Innovationsspielräume. Zwischen beiden Polen erhält sich, bislang zumindest, ein weiterer Bereich, der nur bedingt einem Veränderungsdruck ausgesetzt ist und in dem schwer übergehbare Beharrungskräfte wirksam sind. Festhalten lässt sich, dass das deutsche Insider-dominierte System keinesfalls durchgängig erodiert, vielmehr ist ein Zusammenspiel von Kontinuität und Wandel erkennbar.

Die theoretisch wie politisch spannende Frage ist allerdings, ob damit das deutsche Innovationssystem - wie bisher - auch langfristig zuverlässig in der Lage ist, die für weiteres Wachstum erforderlichen, vor allem international konkurrenzfähigen Technologien zu generieren. Zweifellos verbindet sich mit dem Aufkommen eines kennziffernbasierten und von nicht mit Innovationserfordernissen vertrauten Outsidern gesteuerten Systems ein Erlahmen der bisherigen Innovationsdynamik. Denn das Innovationsdilemma wird hier zugunsten der ökonomischen Kalkulierbarkeit von Innovationen aufgelöst. Die Folge ist, dass die für Außenseiter undurchsichtigen kollektiven Lernprozesse und kreativen Tätigkeiten vor Ort zurückgedrängt werden und die dann rationalisierten Formen der Innovationsorganisation und die Verkürzung von Innovationszyklen trotz teilweise hoher FuE-Ausgaben nur mehr zu "good enough-Lösungen“ und „Scheinlösungen“ führen (Grewer et al. 2007, S. 78). Konkret zeigen sich diese Effekte beispielsweise in der Pharmaindustrie, deren Finanzmarktorientierung zu einer "fundamentalen Beeinträchtigung der Pharmainnovationen" geführt habe (Kädtler 2009, S. 18). Genereller formuliert: Kapital wird „ungeduldiger“ im Hinblick auf die Risiken und die Ungewissheiten technologischer Innovationen und es drängt auf eine schnelle Realisierung von Profiten. Dies gilt insbesondere auch für die verschiedenen Segmente des Marktes für Wagniskapital, obgleich paradoxerweise damit zugleich
Möglichkeiten für in der Vergangenheit kaum finanzierbare FuE-Aktivitäten eröffnet werden. Langfristig dürfte daher der Wandel der Finanzmarktbedingungen Strukturveränderungen im deutschen Innovationssystem induzieren, die auf einen Bedeutungsverlust der bisher zentralen technologisch anspruchsvollen und inkrementellen Innovationen hinauslaufen. Denn solche Innovationsverläufe sind oftmals nicht nur langfristig angelegt; vielmehr weisen sie wegen ihres engen Anwendungs- und Kundenbezugs auch die erwähnten, nur schwer kalkulierbaren „,idiosynkratischen“ Risiken (KfW-Research 2006, S. 108) auf, die von Finanzmarktakteuren gemieden werden. Die bisherige technologisch starke Stellung vieler mittlerer und kleinerer Unternehmen aus industriellen Kernbranchen wie der Investitionsgüterindustrie dürfte dadurch nachhaltig gefährdet werden. Zudem führt die gegenwärtige Finanzkrise zu einer Verschärfung dieser Tendenzen: Der Druck auf die Unternehmen zu weiterer Restrukturierung und Kostenminimierung nimmt $\mathrm{zu}$, die verfügbaren unternehmensinternen Finanzmittel nehmen zumeist massiv ab, während externe Finanzierungsquellen immer schwerer zu erschließen sind. Und es kann keineswegs, auch nicht längerfristig, von einem verringerten Einfluss der finanzmarktorientierten Akteure ausgegangen werden.

\section{LITERATUR}

Achleitner, A.-K./Andres, C./Betzer, A./Weir, C. (2008): Economic Consequences of Private Equity Investments on the German Stock Market, mimeo, University of Bonn, January

Beyer, J. (2006): Vom “kooperativen Kapitalismus" zum Finanzmarktkapitalismus - eine Ursachenanalyse, in: Brinkmann, U./Krenn, K./Schief S. (Hrsg.): Endspiel des kooperativen Kapitalismus?, Wiesbaden, S. 35-57 Briken, K./Kurz, C. (2006): Innovationen in der deutschen Pharma- und Biotechindustrie, in: Heine, H./Schumann, M./Wittke, V. (Hrsg.): Wer den Ast absägt, auf dem er sitzt, kann deshalb noch längst nicht fliegen, Berlin, S. 39-64

Bundesministerium für Bildung und Forschung (BMBF) (Hrsg.) (2007): Bericht zur technologischen Leistungsfähigkeit Deutschlands, Bonn/Berlin Carpenter, M./Lazonick, W./O'Sullivan, M. (2003): The Stock Market and Innovative Capability in the New Economy: The Optical Networking Industry, in: Industrial and Corporate Change 5, S. 963-1034
Caspar, S./Lehrer, M./Soskice, D. (1999): Can High-technology Industries Prosper in Germany? Institutional Frameworks and the Evolution of the German Software and Biotechnology Industries, in: Industry and Innovation 6, S. 5-24

Champenois, C./Engel, D./Heneric, O. (2006): What Kind of Biotechnology Companies do Venture Capitalists and Corporate Investors Prefer?, in: Applied Economics 5, S. 505-518

Deutschmann, C. (2005): Finanzmarkt-Kapitalismus und Wachstumskrise, in: Windolf, P. (Hrsg.): Finanzmarkt-Kapitalismus - Analysen zum Wandel von Produktionsregimen, Kölner Zeitschrift für Soziologie und Sozialpsychologie, Sonderheft 45, S. 58-84

Deutschmann, C. (2008): Kapitalistische Dynamik. Eine gesellschaftstheoretische Perspektive, Wiesbaden

Dosi, G. (1990): Finance, Innovation and Industrial Change, in: Journal of Economic Behavior and Organization 3, S. 299-319 
Expertenkommission Forschung und Innovation (EFI) (Hrsg.) (2009): Gutachten zu Forschung, Innovation und technologischer Leistungsfähigkeit 2009, EFI, Berlin

Fagerberg, J. (2005): Innovation: A Guide to the Literature, in: Fagerberg, J./Mowery, D./Nelson, R. R. (Hrsg.): The Oxford Handbook of Innovation, Oxford, S. 1-27

FAZ (2009): Wagniskapital aus der Konzernkasse , 11.4., S. 17

Franks, J./Mayer, C. (1997): Corporate ownership and control in the U. K., Germany and france, in: Journal of Applied Corporate Finance 4, S. $30-45$

Freye, S. (2009): German Business Elite in Flux. MPIfG Discussion Paper, Max-Planck-Institut für Gesellschaftsforschung, Köln, S. 59-64

Fryges, H./Gottschalk, S./Licht, G./Müller, K. (2007): Hightech-Gründungen und Business Angels, Endbericht für das Bundesministerium für Wirtschaft und Technologie, ZEW Mannheim

Gerybadze, A. (2004), Technologie- und Innovationsmanagement, München

Grewer, H. G./Matthäi, I./Reindl, J. (2007): Der innovative Ältere, München/Mering

Hall, P. A./Soskice, D. (2001): An Introduction to Varieties of Capitalism', in: Hall, P. A./Soskice, D. (Hrsg.): Varieties of Capitalism. The Institutional Foundations of Comparative Advantage, Oxford, S. 1-70

Hirsch-Kreinsen, H. (2008): "Low-Tech“ Innovations, in: Industry and Innovation 1, S. 19-43

Höpner, M. (2003): Wer beherrscht die Unternehmen?, Frankfurt/New York

Huffschmid, J. (1999): Politische Ökonomie der Finanzmärkte, Hamburg Jürgens, U./Sablowski, T. (2008): Sektorale Innovationsprozesse und die Diskussion über deutsche Innovationsschwächen, Düsseldorf

Kädtler, J. (2005): Finanzmärkte: Zur Soziologie einer organisierten Öffentlichkeit, SOFI-Mitteilungen 33, S. 31-37

Kädtler, J. (2009): Finanzialisierung und Finanzmarktrationalität. Zur Bedeutung konventioneller Handlungsorientierungen im gegenwärtigen Kapitalismus, SOFI Working Paper, Nr. 5

Kalkowski, P./Mickler, O./Manske, F. (1995): Technologiestandort Deutschland. Produktinnovationen im Maschinenbau: traditionelle Stärken - neue Herausforderungen, Berlin

Kamp, L. (2007): Zum Einfluss von Private Equity- und Hedge-Fonds auf die Wirtschaft, in: WSI Mitteilungen 11, S. 596-602

KfW-Research (2006): Mittelstands- und Strukturpolitik 37, Sonderband „Innovationen im Mittelstand“, KfW, Frankfurt/M

Klier, D. O./Harrigan, K. R./ Welge, M. K. (2009): Managing Diversified Portfolios, Dortmunder Beiträge zur Unternehmensführung, TU Dortmund

Knorr Cetina, K. /Preda, A. (2006): Introduction, in: Knorr Cetina, K./Preda, A. (Hrsg.): The Sociology of Financial Markets, Oxford, S. 1-15
Lane, C. (2003): Changes in Corporate Governance of German Corporations: Convergence to the Anglo-American Model?, in: Competition \& Change 2-3, S. 79-100

Lazonick, W. (2003): The Theory of the Market Economy and the Social Foundations of Innovative Enterprise, in: Economic and Industrial Democracy 1, S. 9-44

Lazonick, W. (2007): Shareholder value and the governance of innovative enterprise, in: Jürgens, U./Sadowski, D./Schuppert, G. F./Weiss, M. (Hrsg.): Perspektiven der Corporate Governance, Baden-Baden, S. 472500

Lembke, J. (2008): Interesse an Geld, nicht an Farbe und Design, in: FAZ vom 13.5., S. 27

O'Sullivan, M. (2005): Finance and Innovation, in: Fagerberg, J./Mowery, D./Nelson, R. R. (Hrsg.): The Oxford Handbook of Innovation, Oxford, S. 240-265

Rammer, C. (2009): Innovationsverhalten der Unternehmen in Deutschland 2007, Studien zum deutschen Innovationssystem 04, Mannheim et al.

Rammer, C./Zimmermann, V./Müller, E./Heger, D./Aschhoff, B./Reize, R. (2006): Innovationspotenziale von kleinen und mittleren Unternehmen, ZEW Wirtschaftsanalysen, Bd. 79, Baden-Baden

Rammert, W. (1992): Wer oder was steuert den technischen Fortschritt? in: Soziale Welt 1, S. 7-25

Schumpeter, J. (1997): Theorie der wirtschaftlichen Entwicklung, Berlin Springler, E. (2007): Basel II - Finanzierung und wirtschaftliche Entwicklung, in: WSI Mitteilungen 12, S. 664-669

Tylecote, A./Conesa, E. (1999): Corporate Governance, innovation system and industrial performance, in: Industry and Innovation 1, June, S. $25-50$

Tylecote, A./Ramirez, P. (2006): Corporate Governance and Innovation: The UK compared with the US and "insider" economies, in: Research Policy 35, S. 160-180

Tylecote, A./Visintin, F. (2009): Corporate Governance, Finance and the Technological Advantage of Nations, London/New York

Vieweg, H.-G. (2001): Der mittelständische Maschinenbau am Standort Deutschland - Chancen und Risiken im Zeitalter der Globalisierung und "New Economy", mimeo, IFO Institut, München

Werle, R. (2005): Institutionelle Analyse technischer Innovationen, in: Kölner Zeitschrift für Soziologie und Sozialpsychologie 2, S. 308-332 Windolf, P. (2005): Was ist Finanzmarktkapitalismus?, in: Ders. (Hrsg.): Finanzmarkt-Kapitalismus - Analysen zum Wandel von Produktionsregimen, Kölner Zeitschrift für Soziologie und Sozialpsychologie, Sonderheft 45, S. 20-57

Windolf, P. (2008): Eigentümer ohne Risiko: Die Dienstklasse des Finanzmarktkapitalismus, in: Zeitschrift für Soziologie 6, S. 516-535 Wolf, H./Mickler, O./Manske, F. (1992): Eingriffe in Kopfarbeit, Berlin 